\title{
Discovery and identification of potential biomarkers of papillary thyroid carcinoma
}

\author{
Yuxia Fan ${ }^{1}$, Linan Shi ${ }^{2}$, Qiuliang Liu', Rui Dong1, Qian Zhang1, \\ Shaobo Yang ${ }^{1}$, Yingzhong Fan ${ }^{1}$, Heying Yang ${ }^{1}$, Peng $\mathrm{Wu}^{2}$, Jiekai $\mathrm{Yu}^{3}$, \\ Shu Zheng ${ }^{3}$, Fuquan Yang*2 and Jiaxiang Wang*1
}

Address: ${ }^{1}$ Department of Surgery, the First Affiliated Hospital, Zhengzhou University, Zhengzhou, Henan 450052, PR China, ${ }^{2}$ Proteomic Platform, Institute of Biophysics, Chinese Academy of Sciences, Beijing 100101, PR China and ${ }^{3}$ Institute of Cancer, the Second Affiliated Hospital, College of Medicine, Zhejiang University, Hangzhou, Zhejiang 310009, PR China

Email: Yuxia Fan - fanyuxia1981@126.com; Linan Shi - lianli_shi@126.com; Qiuliang Liu - qiuliang_liu@126.com; Rui Dong - dongrui_good@126.com; Qian Zhang - zhang_qian2006@163.com; Shaobo Yang - shao_bo_yang@126.com;

Yingzhong Fan - yingzhong_fan@126.com; Heying Yang - heying_yang@163.com; Peng Wu - peng_wu008@yahoo.cn;

Jiekai Yu - jiekai_yu@yahoo.cn; Shu Zheng - shu_zheng@yahoo.cn; Fuquan Yang* - fuquan_yang@yahoo.cn;

Jiaxiang Wang* - wjiaxiang@zzu.edu.cn

* Corresponding authors

Published: 28 September 2009

Molecular Cancer 2009, 8:79 doi:10.1186/1476-4598-8-79
Received: I March 2009

Accepted: 28 September 2009

This article is available from: http://www.molecular-cancer.com/content/8/I/79

(c) 2009 Fan et al; licensee BioMed Central Ltd.

This is an Open Access article distributed under the terms of the Creative Commons Attribution License (http://creativecommons.org/licenses/by/2.0), which permits unrestricted use, distribution, and reproduction in any medium, provided the original work is properly cited.

\begin{abstract}
Background: Thyroid carcinoma is the most common endocrine malignancy and a common cancer among the malignancies of head and neck. Noninvasive and convenient biomarkers for diagnosis of papillary thyroid carcinoma (PTC) as early as possible remain an urgent need. The aim of this study was to discover and identify potential protein biomarkers for PTC specifically.

Methods: Two hundred and twenty four (224) serum samples with 108 PTC and II6 controls were randomly divided into a training set and a blind testing set. Serum proteomic profiles were analyzed using SELDI-TOF-MS. Candidate biomarkers were purified by HPLC, identified by LC-MS/ MS and validated using ProteinChip immunoassays.

Results: A total of 3 peaks ( $\mathrm{m} / \mathrm{z}$ with $9190,663 \mathrm{I}$ and $8697 \mathrm{Da}$ ) were screened out by support vector machine (SVM) to construct the classification model with high discriminatory power in the training set. The sensitivity and specificity of the model were $95.15 \%$ and $93.97 \%$ respectively in the blind testing set. The candidate biomarker with $\mathrm{m} / \mathrm{z}$ of $9190 \mathrm{Da}$ was found to be up-regulated in PTC patients, and was identified as haptoglobin alpha-I chain. Another two candidate biomarkers (663I, $8697 \mathrm{Da})$ were found down-regulated in PTC and identified as apolipoprotein C-I and apolipoprotein C-III, respectively. In addition, the level of haptoglobin alpha-I chain $(9190 \mathrm{Da})$ progressively increased with the clinical stage I, II, III and IV, and the expression of apolipoprotein C-I and apolipoprotein C-III (663I, $8697 \mathrm{Da}$ ) gradually decreased in higher stages.
\end{abstract}

Conclusion: We have identified a set of biomarkers that could discriminate PTC from non-cancer controls. An efficient strategy, including SELDI-TOF-MS analysis, HPLC purification, MALDI-TOFMS trace and LC-MS/MS identification, has been proved successful. 


\section{Background}

Thyroid carcinoma is the most common endocrine malignancy and a common cancer among the malignancies of head and neck. It comprises $91.5 \%$ of all endocrine malignancies and $1 \%$ of all malignant diseases [1]. An estimated 33550 new cases are diagnosed annually in the United States and recent statistics shows the incidence of thyroid carcinoma has increased, especially in papillary thyroid carcinomas (PTC) [2]. PTC is the most common type, which accounts for $80 \%$ of all thyroid cancers [3]. Early accurate diagnosis and timely treatment are critical for improving long-term survival of PTC patients. Many diagnostic tools have been used for thyroid carcinoma, such as sonography, computed tomography, magnetic resonance imaging, cytological examination and fine-needle aspiration. Currently, although ultrasound-guided fine-needle aspiration biopsy is considered as the most effective test for distinguishing malignant from benign thyroid nodules, its sensitivity is approximately $93 \%$ and its specificity is $75 \%$ [4]. At the same time, researchers have been seeking valuable biomarkers for thyroid carcinoma diagnosis, such as galectin-3, fibronectin-1, CITED1, HBME1, cytokeratin-19 and TPO, and so on. What is disappointing is that all these biomarkers either are lacking specificity to some degree, or have a poor positive predictive value [5-9]. To distinguish a malignant thyroid nodule from a benign lesion more accurately, the diagnostic test, however, still needs to be improved. Moreover, a noninvasive screening method for thyroid malignancy remains unavailable.

Recent advances in the proteomics study have introduced novel techniques for the screening of cancer biomarkers and improved early and accurate diagnosis of cancer diseases to a new horizon [10]. Surfaced enhanced laser desorption/ionization time of flight mass spectroscopy (SELDI-TOF-MS), which generates the protein fingerprint by MS, has been proved a powerful tool for potential biomarker discovery $[11,12]$. Recently, the SELDI-TOFMS analysis has been successfully used to identify specific biomarkers for various cancers, such as ovarian cancer, prostate cancer, pancreatic cancer, colon cancer, breast cancer, etc [13-17]. In search of biomarkers for diagnosing PTC, a few pilot studies based on proteomics were conducted, in which SELDI-TOF-MS has been utilized $[18,19]$. However, no specific protein biomarkers have been identified and validated in those reports.

In this study, firstly, we used SELDI-TOF-MS technology to screen potential protein patterns specific for PTC and then purified the candidate protein biomarker peaks by HPLC, identified by LC-MS/MS and finally confirmed these biomarkers by ProteinChip Immunoassays. To the best of our knowledge, this is the first time that proteins biomarkers have been identified for PTC.

\section{Results}

\section{Serum protein profiles and data processing}

Serum samples from the training set were analyzed and compared by SELDI-TOF-MS with WCX2 chip. All MS data were baseline subtracted and normalized using total ion current, and the peak clusters were generated by Biomarker Wizard software. After carrying out Wilcoxon rank sum tests to determine relative signal strength, 26 peaks with $p$ value $<0.01$ were obtained. Seven protein peaks were found up-regulated and 19 peaks were found down-regulated in PTC group (data not shown). From the random combination of protein peaks with remarkable variation, support vector machine (SVM) screened out the combined model with maximum Youden index of the predicted value, identifying 3 markers positioned at 9190 , 6631 and 8697 respectively. In the PTC group, the 9190 Da protein was remarkably elevated while 6631 \& 8697 Da proteins were significantly decreased (figure 1 ). The descriptive statistics of these 3 markers are shown in Table 1. In addition, the level of 9190 Da protein progressively increased with the clinical stage I, II, III and IV, and the expression of 6631, 8697 Da proteins gradually decreased in higher stages (figure 2). Combining 3 potential markers, using the method of leave-1-out for cross detection, the sensitivity of discriminating 60 PTC and 40 normal subjects was $98 \%$, and its specificity was $97 \%$.

\section{Protein peak validation}

The remaining 48 PTC and 76 control serum samples (20 healthy controls and 56 patients with benign thyroid node) as a blind testing set, were analyzed to validate the accuracy and validity of the classification model derived from the training set. The descriptive statistics of the three markers in 48 PTC patients and 76 non-cancer controls are shown in Table 2. The classification model distinguished the PTC samples from controls with a sensitivity of $95.15 \%$, specificity of $93.97 \%$, and positive predictive value of $96.0 \%$, respectively. The area under the receiver operating characteristics (ROC) curve of this model was 0.971 .

\section{Purification and identification of candidate protein biomarkers}

Serum samples from PTC patients were used for the purification of the up-regulated candidate protein biomarker (9190 Da), and serum samples from healthy controls

Table I: The relative peak intensity of three distinct protein spectra found in sera of PTC patients.

\begin{tabular}{llll}
\hline $\boldsymbol{m} / \mathbf{z}$ & $\begin{array}{l}\text { Patients with PTC } \\
\text { (mean } \pm \text { SD) }\end{array}$ & $\begin{array}{l}\text { Healthy Individuals } \\
(\text { mean } \pm \text { SD) }\end{array}$ & $\boldsymbol{P}$ \\
\hline 9190 & $6853.82 \pm 1585.23$ & $282.46 \pm 118.09$ & 0.000538 \\
6631 & $2706.56 \pm 578.17$ & $4697.16 \pm 989.85$ & 0.001381 \\
8697 & $3017.98 \pm 600.28$ & $4924.32 \pm 1048.11$ & 0.001672
\end{tabular}




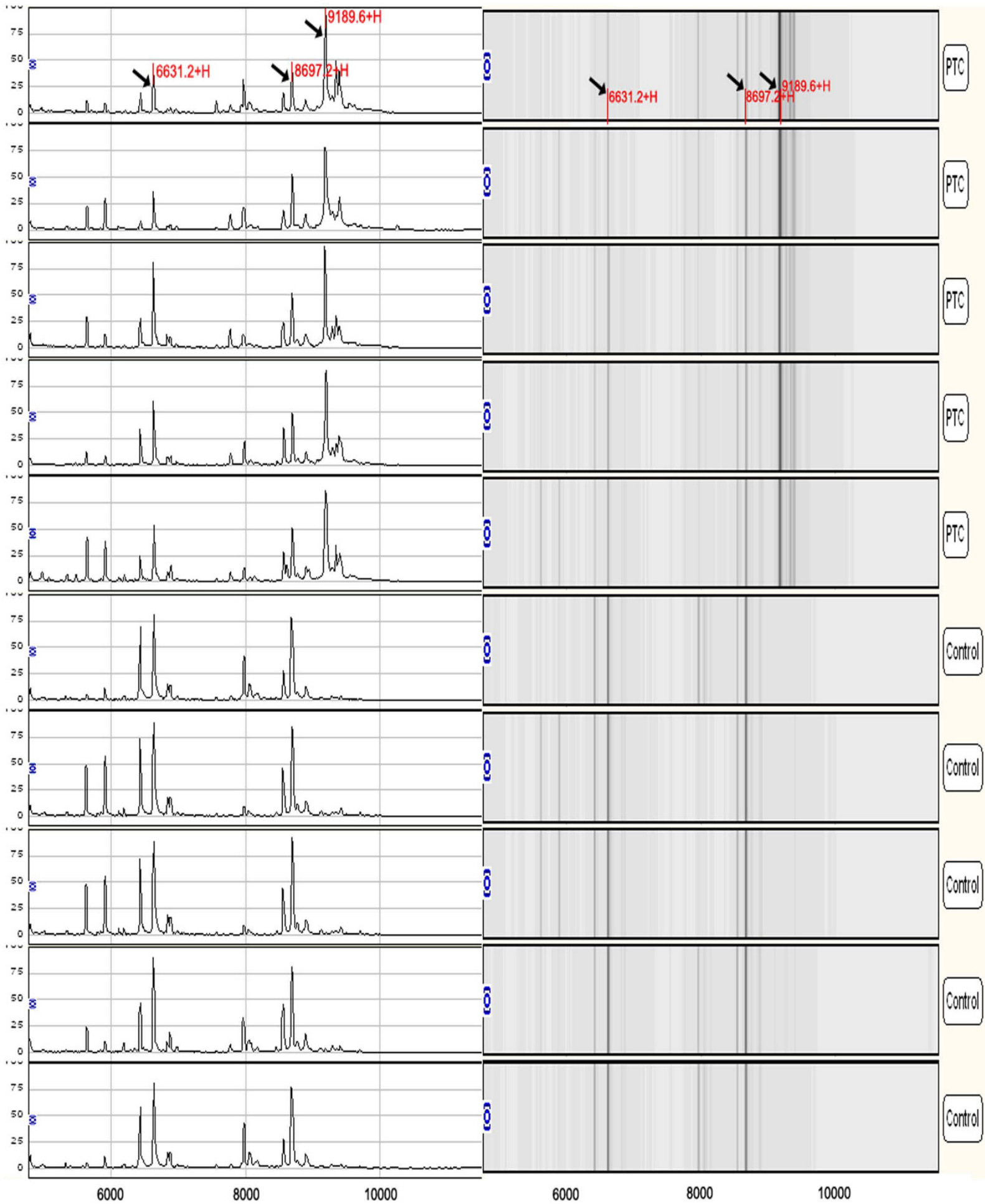

Figure I

A representative mapping of SELDI-TOF-MS analysis of sera from PTC patients and healthy controls. Differentially expressed proteins with potential diagnostic significance are arrowed. Top-group denotes sera from patients with PTC, in which the protein with $\mathrm{m} / \mathrm{z}$ of $9190 \mathrm{Da}$ was over-expressed. Bottom-group denotes sera from healthy individuals, in which the proteins with $\mathrm{m} / \mathrm{z}$ of $663 \mathrm{I}$ and $8697 \mathrm{Da}$ were up-regulated. 


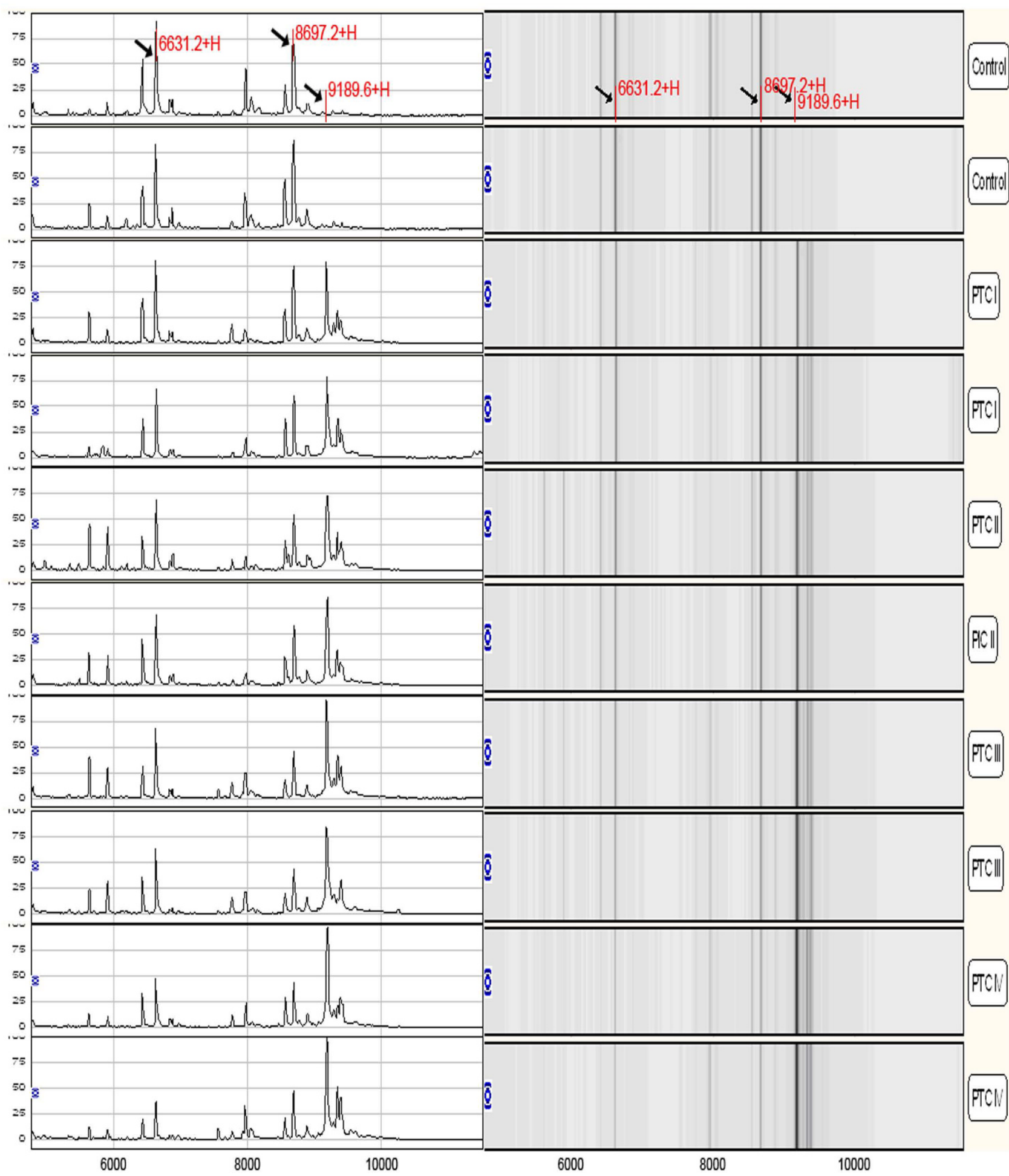

Figure 2

A representative mapping of SELDI-TOF-MS analysis from different stages of PTC patients and non-cancer controls. The level of the $9190 \mathrm{Da}$ protein progressively increased with the clinical stage I, II, III and IV, and the expression of $663 \mathrm{I}$ and $8697 \mathrm{Da}$ proteins gradually decreased in higher stages. 
Table 2: The descriptive statistics of these 3 markers in the blind testing set.

\begin{tabular}{llll}
\hline $\boldsymbol{m} / \mathbf{z}$ & $\begin{array}{l}\text { Patients with PTC } \\
\text { (mean } \pm \text { SD) }\end{array}$ & $\begin{array}{l}\text { Non-cancer Controls } \\
\text { (mean } \pm \text { SD) }\end{array}$ & $\boldsymbol{p}$ \\
\hline 9190 & $6798.89 \pm 1517.76$ & $271.35 \pm 102.04$ & 0.000526 \\
6631 & $2786.03 \pm 596.11$ & $4705.23 \pm 991.45$ & 0.001402 \\
8697 & $3011.62 \pm 598.13$ & $4912.36 \pm 1042.79$ & 0.001669
\end{tabular}

were used for the purification of the two down-regulate proteins $(6631,8697 \mathrm{Da})$ using WCX SPE and C18 HPLC. Figure 3 shows the results of MALDI-TOF-MS analysis of the three purified candidate protein biomarkers.

After digestion with modified trypsin, the peptide mixture was analyzed by nano-LC-MS/MS. Figure 4 shows the results of the LC-MS/MS chromatogram (A) and MS/MS spectrum of one identified peptide (B) from protein (8697 Da). Table 3 shows the results of identification of the three candidate protein biomarkers. They were haptoglobin alpha-1 chain (9190 Da) [NCBI:P00738], apolipoprotein C-I (6631 $\mathrm{Da})$ [NCBI:P02654] and apolipoprotein C-III (8697 Da) [NCBI:CAA25233]. The whole sequence of the three candidate protein markers is given by combination of high sequence coverage and accurate molecular weight (MW) measurement using MALDI-TOF-MS.

\section{Validation of three candidate protein biomarkers}

A ProteinChip-array-based immunoassay (Ciphergen Biosystems) was used to specifically capture haptoglobin alpha-1 chain, apolipoprotein C-I and apolipoprotein CIII from crude serum samples and to confirm the significance of each marker. The anti-haptoglobin alpha-chain antibody specifically captured the previously identified 9190 Da protein. The anti-apolipoprotein C-I array was developed to capture apolipoprotein C-I (6631 Da) and the apolipoprotein C-III antibody against specifically captured apolipoprotein C-III (8697 Da). (Figure 5)

\section{Discussions}

In this study, we obtained serum protein mass spectra from PTC patients and controls using SELDI-TOF-MS. Based on the serum proteomic profiles, we constructed a classification model to discriminate PTC patients from non-cancer controls. One of the challenges in the analysis of SELDI-TOF-MS-generated data is to reduce the false protein peaks, in which the discriminatory power is due to random variation [20]. To solve this problem in the data processing of this experiment, we eliminated noise by discrete wavelength, identified mass-charge peaks of specimens using the method of local extremum, and clustered mass-charge peaks by setting $10 \%$ as the minimum threshold. Wilcoxon rank sum test analysis assessed the relative importance of each peak in the discrimination of 2 kinds of specimen according to $P$ values. Furthermore,
SVM was employed in our experiment, which is a kind of classification technology proposed by Vapnik and others. In the model discrimination, the popularization, model selection, overfitting, latitude disaster, and other problems of the small specimen model have been solved successfully in SVM [21-23]. The procedures included randomly combining the remarkably different masscharge peaks and inputting them into SVM, screening out the markers, building the discrimination model, and then using the method of leave one out to assess the model by means of cross verification. By combination among these procedures mentioned above, the popularization of the model building and the accuracy of the prediction were ensured. The classification model could discriminate patients with PTC from non-cancer controls with a sensitivity of $95.15 \%$ and a specificity of $93.97 \%$ in the blind testing set. The up-regulated candidate protein biomarker was identified as haptoglobin alpha-1 chain (9190 Da). Another two down-regulated candidate protein biomarkers (6631 and $8697 \mathrm{Da}$ ) were identified as apolipoprotein C-I and apolipoprotein C-III.

Among the proteins identified by LC-MS/MS, haptoglobin alpha-1 chain was significantly elevated in PTC patients and this protein may play a critical role in the development of PTC. Intact haptoglobin, composed of two different polypeptides (alpha and beta-chains), is an acute phase protein capable of binding haemoglobin and preventing iron loss [24]. It was reported that body iron could promote neoplastic cell growth and accumulate in cancer cells more than in normal cells [25]. Furthermore, a few other studies have demonstrated that there is a higher cancer risk in patients with larger iron stores than those with small iron stores [26]. Collectively, highly expressed haptoglobin results in a high hemoglobin/iron existence and raises the possibility of a causative involvement of iron-derived oxidative stress in the tumour development. Recently, there are several reports in the literature showing increased expression of haptoglobin in ovarian cancer, prostatic carcinoma and pancreatic cancer [27-29], and the level of haptoglobin alpha chain up-regulated in serum of breast cancer [30]. However, it is necessary to understand more clearly of the role of haptoglobin alpha1 chain in the development of PTC through further studies of biological mechanisms of thyroid carcinoma.

Apolipoproteins (APOs) are lipid carriers and previous studies about APO mainly focused on lipoprotein metabolism. Recently, APOs have been reported to regulate many cellular function. For example, the protein kinase Akt can be elicited by APO C-I, which in turn promotes growth factor-mediated cell survival and block apoptosis [31]. In this study, the APO C-I is down-regulated in the serum of PTC patients, which indicates that APO C-I may be related to PTC. Thus, besides the function of APO C-I in lipid metabolism, additional function of APO C-I in 

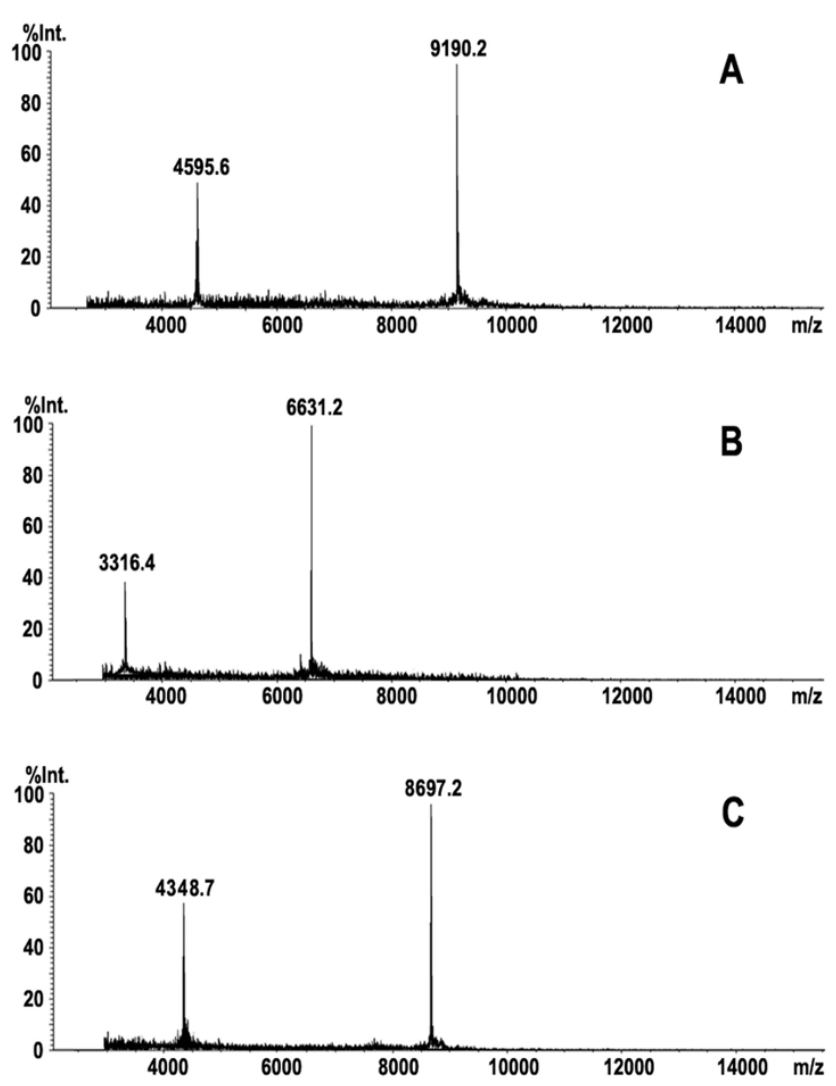

Figure 3

MALDI-TOF-MS spectra of three purified potential protein markers.

cancerogenesis may also exist. However, the mechanism of how APO C-I is degraded in PTC is not very clear, and further research is required. APO C-III is primarily synthesized in the liver and in a minor degree in the small intestine. The regulatory elements that control both hepatic and intestinal transcription of the human APO C-III gene are localized between nucleotides -792 and -25 of the APO C-III promoter. The mechanism of why APO C-III was decreased in PTC may be as follows. Orphan members of the nuclear hormone receptor superfamily can bind to the hormone response elements (HREs), and strongly enhance or repress APO C-III promoter's activity. It was reported that a combination of RXRalpha and T3Rbeta could repress the APO C-III promoter's activity in the presence of T3 [32]. In addition, the thyroid stimulating hormone (TSH) level was significantly higher in PTC than in non-cancer control [33], correspondingly, T3 increased, thus the expression of APO C-III was possibly inhibited in PTC patients.

\section{Conclusion}

In summary, we have identified a set of protein peaks that could discriminate PTC from non-cancer controls. From the protein peaks specific for PTC disease, we identified haptoglobin alpha-1 chain, apolipoprotein C-I and apolipoprotein C-III as potential proteomic biomarkers of PTC. Further studies with larger sample sizes will be needed to verify the specific protein markers. An efficient strategy, composed of SELDI-TOF-MS analysis, HPLC purification, MALDI-TOF-MS trace and LC-MS/MS identification has been proved very successful.

\section{Materials and methods \\ Patients and serum samples}

Serum samples were obtained from 224 individuals with informed consent in the Department of General Surgery, the First Affiliated Hospital of Zhengzhou University. These 224 individuals included 108 patients with PTC, 56 patients with benign thyroid node, and 60 healthy individuals. Patients with PTC had a median age of 43 years (ranging from 23 to 75 years, 27 men and 81 women), and the sera was obtained at the time of diagnosis. All 108 patients were distributed in 4 stages according to UICC. In stage I there were 85 patients, stage II, III \& IV consisted of $12,8 \& 3$ patients respectively. The diagnosis, stage and other information about these patients are listed in Table 4 and Table 5. Inclusion criteria for the study were patients with a confirmed diagnosis of PTC, the ability to provide written, informed consent, and without any previous treatment. Patients with benign thyroid node and healthy donors were defined as controls in this study. The benign thyroid node group and the healthy individual group were age- and gender-matched with the PTC group. Pathological diagnosis of all the PTC and benign thyroid nodes were confirmed independently by two pathologists. All serum samples were collected preoperatively in the morning before breakfast. The sera were left at room temperature for $1 \mathrm{~h}$, centrifuged at $3000 \mathrm{rpm}$ for $10 \mathrm{~min}$, and then stored at $-80^{\circ} \mathrm{C}$.

\section{Reagents and instruments}

Sinapinic acid (SA) was purchased from Fluka (USA). ProteinChip Biosystems (Ciphergen PBS II plus SELDITOFMS) and WCX2 chip were purchased from Ciphergen Biosystems (USA). All other SELDI-TOF-MS related reagents were acquired from Sigma (USA). Ziptip C18 was purchased from Millipore (USA). Trypsase was purchased from Promega (USA). IAM was purchased from AppliChem (GER). DTT was purchased from BIO-RAD (GER). MALDI-TOF-MS was purchased from Kratos Analytical Co (UK) and HPLC was purchased from Shimadzu (JPN). LC-MS/MS was purchased from Thermo Electron Corporation (USA).

\section{SELDI-TOF-MS analysis of serum protein profiles}

Protein profiling of serum samples was determined by SELDI-TOF-MS using the WCX2 (weak cation exchange) Proteinchip arrays (Ciphergen Biosystems, USA). Frozen serum samples were defrosted on ice and spun at 10000 
Table 3: Identification of the three potential protein biomarkers with identified peptides and covered sequence.

\begin{tabular}{|c|c|c|c|}
\hline $\mathbf{m} / \mathbf{z}$ & Protein Name & Peptides Identified & Sequence* \\
\hline \multirow[t]{7}{*}{9190} & Haptoglobin $\alpha$-chain & K.LRTEGDGVYTLNNEK.Q & $\begin{array}{l}\text { addgcpkppeiahgyvehsvryqck nyyklrtegdgvytInnekqwinka } \\
\text { vgdklpeceavcgkpknpanpvq }\end{array}$ \\
\hline & & R.YQCKNYYK.L & \\
\hline & & K.AVGDKLPECEAVCGK.P & \\
\hline & & K.QWINKAVGDK.L & \\
\hline & & R.TEGDGVYTLNNEK.Q & \\
\hline & & K.LPECEAYCGKPK.N & \\
\hline & & K.PKNPANPVQ.- & \\
\hline \multirow[t]{7}{*}{6631} & Apolipoprotein C-I & G.TPDVSSALDK.L & tpdvssaldklkefgntledkarelis rikqselsakmrewfsetfqkvkeklkids \\
\hline & & K.EFGNTLEDKAR.E & \\
\hline & & K.LKEFGNTLEDK.A & \\
\hline & & R.EWFSETFQK.V & \\
\hline & & G. TPDVSSALDKLK.A & \\
\hline & & K.MREWFSETFQK.V & \\
\hline & & K.ARELISRIK.Q & \\
\hline \multirow[t]{6}{*}{8697} & Apolipoprotein C-III & K.DALSSVQESQVAQQAR.G & $\begin{array}{l}\text { seaedasllsfmqgymkhatktakdalssvq esqvaqqargwvtdgfsslkdywstvkd } \\
\text { kfsefwdldpevrptsavaa }\end{array}$ \\
\hline & & R.GWVTDGFSSLK.D & \\
\hline & & K.TAKDALSSVQESQVAQQAR.G & \\
\hline & & R.GWVTDGFSSLKDYWSTVK.D & \\
\hline & & K.DKFSEFWDLDPEVR.P & \\
\hline & & K.DKFSEFWDLDPEVRPTSAVAA.- & \\
\hline
\end{tabular}

*: Bold letters show the covered sequence by identified peptide

rpm for 5 min at $4{ }^{\circ} \mathrm{C}$. Each serum sample $(10 \mu \mathrm{l})$ was denatured by addition of $20 \mu \mathrm{l}$ of $\mathrm{U} 9$ buffer ( $9 \mathrm{M}$ urea, $2 \%$ CHAPS, 50 mM Tris-HCI, 1\% DTT, PH 9.0) and vortexed at $4{ }^{\circ} \mathrm{C}$ for $30 \mathrm{~min}$. Each sample was then diluted in 108 $\mu \mathrm{l}$ of low stringency buffer ( $0.1 \mathrm{M}$ sodium acetate, $\mathrm{PH} 4.0)$ and $100 \mu \mathrm{l}$ of each diluted serum sample was hybridized with WCX2 proteinchip arrays, which was held by a bioprocessor (Ciphergen Biosystems) and preactivated twice with $150 \mu \mathrm{l}$ low stringency buffer at room temperature for $5 \mathrm{~min}$. The diluted serum sample was added on the surface of the WCX2 chip for 60 min at room temperature. Each spot was then washed three times with appropriate buffers of various PHs and ionic strengths to eliminate non-adsorbed proteins. After drying the array surface in the air, $1 \mu \mathrm{l}$ saturated sinapinic acid (SA) matrix in 50\% ACN and $0.5 \%$ TFA was applied and allowed to dry. MS analysis was performed on a PBS-II ProteinChip reader (Ciphergen Biosystems). Mass peak detection was analyzed using ProteinChip Biomarker Software version 3.1 (Ciphergen Biosystems). The mass spectra of the proteins were generated using an average of 140 laser shots at a laser intensity of 170 arbitrary units and detector sensitivity was set at 6 . For data acquisition of low-molecularweight proteins, the optimize detection mass range was set from 2 to $20 \mathrm{kDa}$ for all study sample profiles. The instrument was calibrated by the All-in-one peptide molecular mass standard (Ciphergen Biosystems).

\section{Bioinformatics and biostatistics}

Patients with PTC were split into a training set and a blind testing set. Sixty samples of PTC patients (45 stage I, 8 stage II, 4 stage III, 3 stage IV) and 40 healthy controls were selected for a training sample set randomly. To evaluate the accuracy and validity of the classification model, the remaining 48 samples of PTC patients (40 stage I, 4 stage II, 4 stage III) and 76 controls (20 healthy controls and 56 patients with benign thyroid node) were selected for a blind testing set. (Table 6).

The first step of data analysis was to use the undecimated discrete wavelet transform (UDWT) method to denoise the signals. Secondly, the spectra were subjected to baseline correction by aliging with a monotone local minimum curve and mass calibration. The proteomic peaks were detected and quantified by an algorithm that takes the maximal height of every denoised, baseline-corrected, and calibrated mass spectrum into account. Thirdly, the peaks were filtered to maintain a $\mathrm{S} / \mathrm{N}$ of more than three. The $S / N$ of a peak is the ratio of the height of the peak above the baseline to the wavelet-defined noise. Finally, to match peaks across spectra, we pooled the detected peaks if the relative difference in their mass sizes was not more than $0.3 \%$. The minimal percentage of each peak, appearing in all the spectra, is specified to ten. The matched peak across spectra is defined as a peak cluster. If a spectrum does not have a peak within a given cluster, the 

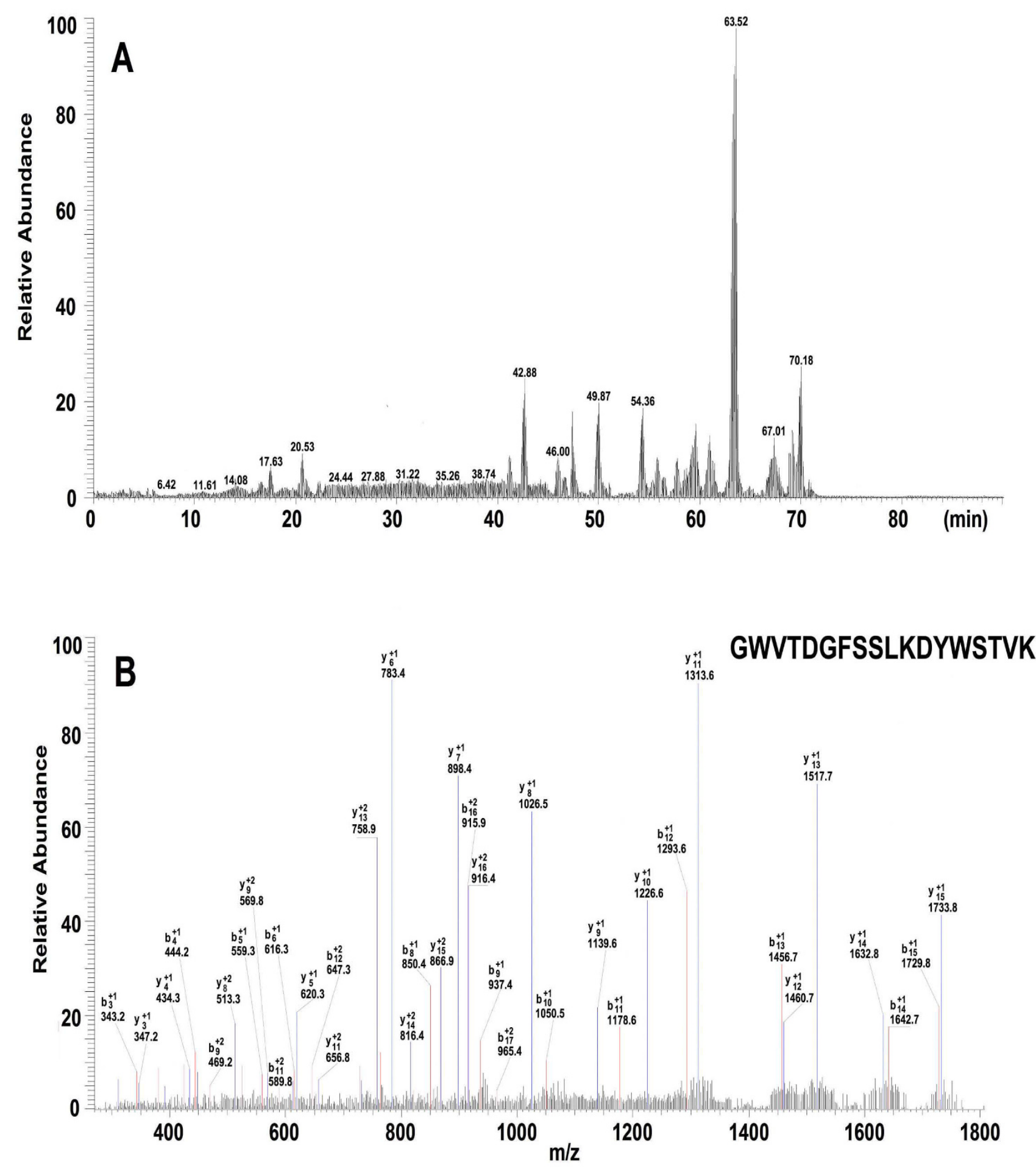

Figure 4

Results of the identification of protein (8697 Da) by LC-MS/MS. (A) Chromatogram of peptide mixture. (B) MS/MS spectrum of one peptide. 

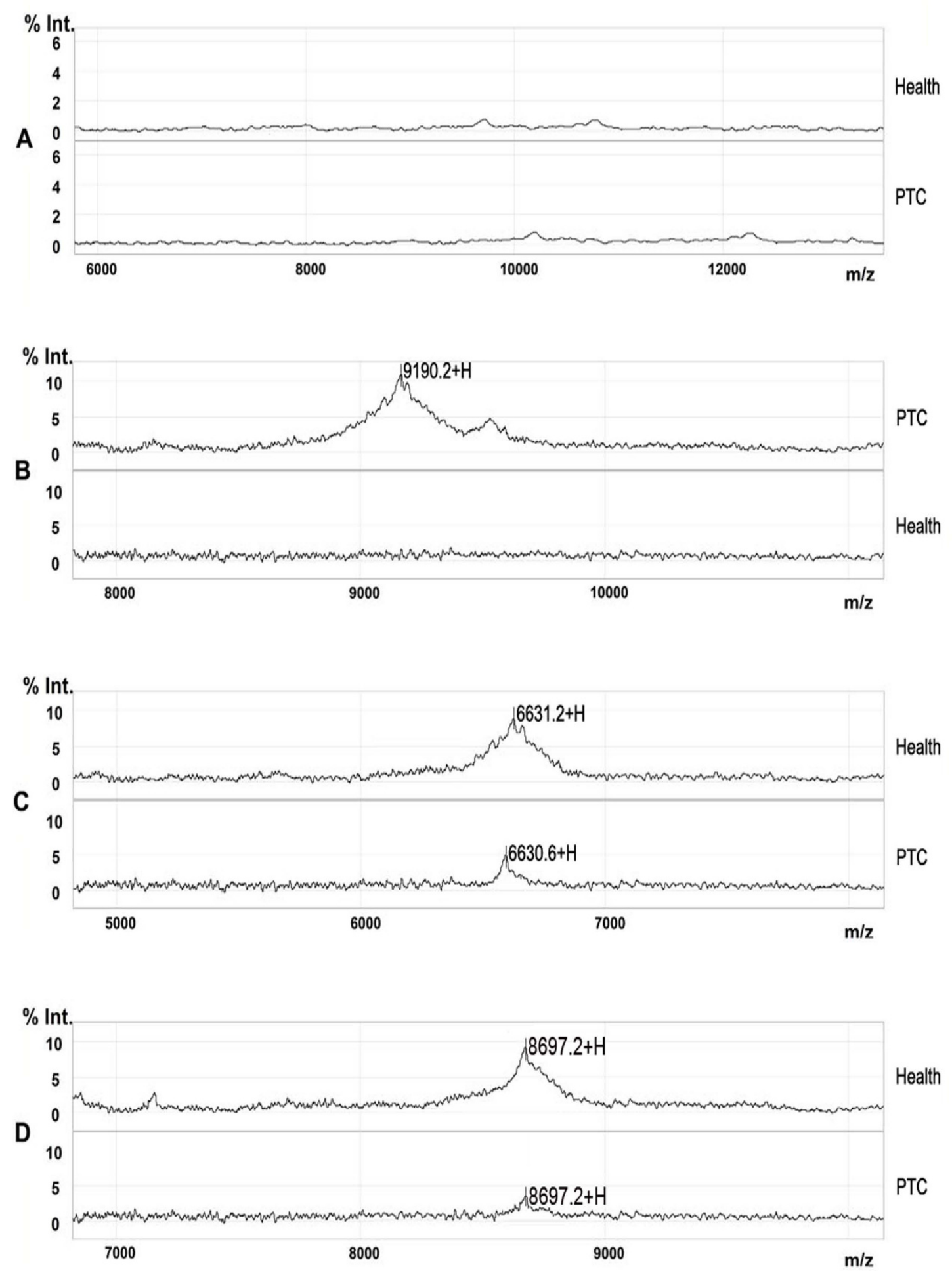

\section{Figure 5}

Representative spectra from ProteinChip array with specific immobilized antibodies. (A) Representative spectra of the negative control (nonspecific rabbit $\lg G$ ). (B) Representative spectra from ProteinChip array with anti-haptoglobin alpha chain antibody. (C) Representative spectra from ProteinChip array with anti-apolipoprotein C-I. (D) Representative spectra from ProteinChip array with anti-apolipoprotein C-III. 
Table 4: The diagnosis, staging and other information about PTC patients in the training set.

\begin{tabular}{|c|c|c|c|c|c|c|c|}
\hline Patient ID & Age (years) & $\begin{array}{l}\text { Sex } \\
(F=\text { female } \\
M=\text { male })\end{array}$ & Diagnosis & Stage & Tumor size $(\mathrm{cm})$ & $\begin{array}{l}\text { Cervial lymph } \\
\text { node metastasis }\end{array}$ & $\begin{array}{l}\text { Distant } \\
\text { metastases }\end{array}$ \\
\hline \multicolumn{8}{|c|}{ Patients $<45$ years } \\
\hline P38 & 23.01 & $\mathrm{~F}$ & PTC & I & 0.5 & None & NO \\
\hline P78 & 29.98 & $\mathrm{~F}$ & PTC & 1 & 1.0 & None & NO \\
\hline P65 & 32.12 & $\mathrm{~F}$ & PTC & I & 1.0 & None & NO \\
\hline P8I & 36.45 & $\mathrm{~F}$ & PTC & I & 0.8 & None & NO \\
\hline P36 & 38.50 & $\mathrm{~F}$ & PTC & I & 0.5 & None & NO \\
\hline P43 & 39.56 & $\mathrm{~F}$ & PTC & I & 1.0 & None & NO \\
\hline PI6 & 40.05 & $\mathrm{~F}$ & PTC & 1 & 0.8 & None & NO \\
\hline P44 & 40.11 & $M$ & PTC & I & 1.0 & Yes(VI lymph node) & NO \\
\hline P37 & 40.25 & $\mathrm{~F}$ & PTC & 1 & 1.6 & None & NO \\
\hline P5 & 40.36 & $\mathrm{~F}$ & PTC & I & 1.0 & None & NO \\
\hline PI8 & 40.50 & $M$ & PTC & I & 0.6 & None & NO \\
\hline P80 & 40.81 & $M$ & PTC & I & 0.7 & None & NO \\
\hline P93 & 40.90 & $\mathrm{~F}$ & PTC & 1 & 1.0 & None & NO \\
\hline P67 & 41.00 & $\mathrm{~F}$ & PTC & I & 1.0 & None & NO \\
\hline P46 & 41.08 & $\mathrm{~F}$ & PTC & I & 1.5 & None & NO \\
\hline P9 & 41.10 & M & PTC & 1 & 1.8 & Yes(VI lymph node) & NO \\
\hline P35 & 41.26 & $M$ & PTC & I & 1.0 & None & NO \\
\hline P47 & 41.38 & $\mathrm{~F}$ & PTC & 1 & 0.8 & None & NO \\
\hline $\mathrm{PI3}$ & 41.44 & $\mathrm{~F}$ & PTC & 1 & 1.0 & None & NO \\
\hline P6 & 41.52 & $M$ & PTC & I & 0.6 & None & NO \\
\hline PI7 & 41.55 & $\mathrm{~F}$ & PTC & 1 & 1.5 & Yes(VI lymph node) & NO \\
\hline P40 & 41.62 & $\mathrm{~F}$ & PTC & I & 0.8 & None & NO \\
\hline P64 & 41.71 & $\mathrm{~F}$ & PTC & I & 0.8 & None & NO \\
\hline PIII & 41.89 & $M$ & PTC & 1 & 1.3 & None & NO \\
\hline P69 & 42.14 & $M$ & PTC & 1 & 1.2 & None & NO \\
\hline P2 & 42.25 & $\mathrm{~F}$ & PTC & 1 & 1.6 & Yes(VI lymph node) & NO \\
\hline $\mathrm{PI} 2$ & 42.36 & $\mathrm{~F}$ & PTC & 1 & 0.8 & None & NO \\
\hline P7 & 42.56 & $\mathrm{~F}$ & PTC & I & 0.8 & None & NO \\
\hline P4 & 42.73 & $M$ & PTC & I & 0.6 & None & NO \\
\hline P49 & 43.20 & $\mathrm{~F}$ & PTC & 1 & 1.2 & None & NO \\
\hline P8 & 43.66 & $M$ & PTC & i & 1.5 & Yes(VI lymph node) & NO \\
\hline P70 & 43.80 & $\mathrm{~F}$ & PTC & I & 0.7 & None & NO \\
\hline P42 & 44.10 & $\mathrm{~F}$ & PTC & I & 1.5 & None & NO \\
\hline PII4 & 44.30 & $M$ & PTC & 1 & 0.8 & None & NO \\
\hline P83 & 44.45 & $\mathrm{~F}$ & PTC & 1 & 1.3 & None & NO \\
\hline P99 & 44.60 & $M$ & PTC & 1 & 0.6 & None & NO \\
\hline \multicolumn{8}{|c|}{ Patients $>45$ years } \\
\hline PI05 & 45.05 & $\mathrm{~F}$ & PTC & 1 & 0.8 & None & NO \\
\hline P7I & 45.58 & $M$ & PTC & II & 2.5 & None & NO \\
\hline PI07 & 45.75 & $M$ & PTC & 1 & 0.7 & None & NO \\
\hline $\mathrm{P} 60$ & 45.81 & $\mathrm{~F}$ & PTC & II & 2.5 & None & NO \\
\hline PIO & 46.48 & $\mathrm{~F}$ & PTC & I & 0.8 & None & NO \\
\hline PIO4 & 46.40 & $\mathrm{~F}$ & PTC & II & 2.3 & None & NO \\
\hline $\mathrm{PI} 4$ & 46.90 & $\mathrm{~F}$ & PTC & II & 2.5 & None & NO \\
\hline P52 & 47.10 & $\mathrm{~F}$ & PTC & 1 & 0.7 & None & NO \\
\hline PII & 47.64 & $M$ & PTC & III & 3.5 & Yes(VI lymph node) & NO \\
\hline P88 & 47.89 & $\mathrm{~F}$ & PTC & I & 0.6 & None & NO \\
\hline P22 & 48.50 & $\mathrm{~F}$ & PTC & I & 0.8 & None & NO \\
\hline P73 & 48.81 & $M$ & PTC & 1 & 0.7 & None & NO \\
\hline P87 & 49.56 & $\mathrm{~F}$ & PTC & III & 4.5 & Yes(VI lymph node) & NO \\
\hline $\mathrm{P} 100$ & 49.89 & $\mathrm{~F}$ & PTC & $\|$ & 2.8 & None & NO \\
\hline $\mathrm{P} 25$ & 50.25 & $\mathrm{~F}$ & PTC & III & 3.8 & Yes(VI lymph node) & NO \\
\hline P85 & 50.89 & $\mathrm{~F}$ & PTC & I & 0.7 & None & NO \\
\hline PIOI & 57.14 & $M$ & PTC & ॥ & 2.5 & None & NO \\
\hline P32 & 58.21 & $\mathrm{~F}$ & PTC & II & 2.3 & None & NO \\
\hline $\mathrm{P} 103$ & 60.10 & $\mathrm{~F}$ & PTC & I & 0.8 & None & NO \\
\hline P53 & 61.12 & $\mathrm{~F}$ & PTC & II & 2.5 & None & NO \\
\hline
\end{tabular}


Table 4: The diagnosis, staging and other information about PTC patients in the training set. (Continued)

\begin{tabular}{llllllll}
\hline P23 & 65.84 & F & PTC & IV & 4.0 & Yes(hibateral) & $\begin{array}{l}\text { Yes } \\
\text { (pulmonary } \\
\text { metastasis) }\end{array}$ \\
P54 & 68.19 & F & PTC & IV & 3.5 & Yes(mediastinal) & $\begin{array}{l}\text { Yes } \\
\text { (osseous metastasis) }\end{array}$ \\
P24 & 72.31 & F & PTC & III & 3.6 & None & No \\
P63 & 74.98 & F & PTC & IV & 3.5 & Yes(hibateral) & Yes \\
& & & & & & & (pulmonary \\
& & & & & & & metastasis) \\
\hline
\end{tabular}

maximal height within the cluster will be assigned to its peak value. The normalization was performed only with the identified peak clusters.

To distinguish between data of different groups, we used a nonlinear SVM classifier, originally developed by Vladimir Vapnik, with a radial-based function kernel, a parameter Gamma of 0.6, and a cost of the constrain violation of 19 . The leave-one-out crossing validation approach was applied to estimate the accuracy of this classifier. The capability of each peak in distinguishing data of different groups was estimated by the $p$ value of Wilcoxson $T$-test. The $p$ value was set at 0.01 to be statistically significant. The remaining 48 samples of PTC patients and 76 controls (20 healthy controls and 56 patients with benign thyroid node), were analyzed to test the classification model. PTC and control samples were then discriminated based on their proteomic profile characteristics. The sensitivity was defined as the probability of predicting PTC cases, the specificity was defined as the probability of predicting control samples. The positive predictive value was defined as the probability of PTC if a test result was positive.

\section{Serum fractionation}

Serum samples both from healthy controls and PTC patients were selected for the purification of the three candidate protein biomarkers. The serum sample was mixed with U9 buffer $(1: 2, \mathrm{v} / \mathrm{v})$ and incubated for $30 \mathrm{~min}$ at room temperature. The sample was then diluted in $5 \mathrm{~mL}$ WCX binding buffer (50 mM NaAc, pH 4.0) and loaded to the CM Ceramic Hyper D WCX SPE column $(6 \times 10$ $\mathrm{mm}$, Pall Life science, USA). After washing with $2 \mathrm{~mL}$ of WCX binding buffer, the column was eluted with $5 \mathrm{ml}$ of eluting buffer ( $2 \mathrm{M} \mathrm{NaCl}, 50 \mathrm{mM} \mathrm{NaAc}, \mathrm{pH} 4.0$ ) at a flow rate of $0.5 \mathrm{ml} / \mathrm{min}$. The eluted fraction was further purified using HPLC.

\section{Purification of candidate protein markers using HPLC}

HPLC separation was performed using SCL-10AVP (Shimadzu, Japan) with a Sunchrom C18 column $(250 \times 4.6$ mm, $5 \mu \mathrm{m}$ particle size, $300 \AA$ ) (The Great Eur-Asia SciTech Development Co. Ltd, Beijing, China) and a C18 guard column $(10 \times 3 \mathrm{~mm}$, Shimadzu, Japan $)$. The mobile phase consisted of solvent A (5\% ACN, 0.1\% TFA) and solvent B (90\% ACN, 0.1\% TFA). The HPLC separation was achieved with a linear solvent gradient: $100 \% \mathrm{~A}(0$ $\min )-15 \%$ B (15 min)-65\% B (65 min)-100\% B (100 $\mathrm{min}$ ) at a flow-rate of $0.5 \mathrm{ml} / \mathrm{min}$. The eluate was detected at multiple wavelengths of 214, 254, $280 \mathrm{~nm}$. Each peak fraction was collected and concentrated using SpeedVac, and then analyzed using AXIMA-CFRTM plus MALDI-TOF mass spectrometer (Shimadzu/Kratos, Manchester, UK) in linear mode to trace the candidate protein biomarkers with $\alpha$-cyano-4-hydrorycinnamic acid (CHCA) as matrix.

\section{Identification of candidate protein biomarkers by LC-MSI MS}

In-solution digestion of each concentrated fraction, which contains one candidate protein biomarker, was performed with a standard protocol. Briefly, each fraction was dissolved in $25 \mathrm{mM} \mathrm{NH}_{4} \mathrm{HCO}_{3}$, and reduced with 10 mM DTT for 1 hour, alkylated by $40 \mathrm{mM}$ iodoacetamide in the dark for $45 \mathrm{~min}$ at room temperature, and then 40 mM DTT was added to quench the iodoacetamide for 30 min at room temperature. Then proteins were proteolysed with $20 \mathrm{ng}$ of modified trypsin (Promega, Madison, WI) in $25 \mathrm{mM} \mathrm{NH}_{4} \mathrm{HCO}_{3}$ overnight at $37^{\circ} \mathrm{C}$. The supernatant was collected and peptides were further extracted in $0.1 \%$ acetic acid and $60 \%$ acetonitrile. Peptide extracts were vacuumdried and resuspended in $20 \mu \mathrm{l}$ of water for mass analysis. Protein digests obtained above were loaded onto a home-made $\mathrm{C} 18$ column $(100 \mathrm{~mm} \times 100 \mu \mathrm{m})$ packed with Sunchron packing material (SP-120-3-ODS-A, $3 \mu \mathrm{m}$ ) and followed with nano-LC-ESI-MS/MS analysis. The LTQ mass spectrometer was operated in a data-dependent mode, in which the initial MS scan recorded the $m / z$ ratios of ions over the mass range from 400-2000 Da firstly, and then the five most abundant ions were automatically selected for subsequent collision-activated dissociation. All MS/MS data were searched against a human protein database downloaded from NCBI using the SEQUEST program (Thermo, USA).

\section{Confirmation of candidate protein biomarkers using ProteinChip Immunoassays}

To confirm the identity of the candidate protein biomarkers, all samples from the initial experiments were reanalyzed by using ProteinChip immunoassays (Ciphergen Biosystems). Specific antibody arrays were prepared by 
Table 5: The diagnosis, staging and other information about PTC patients in the blind testing set.

\begin{tabular}{|c|c|c|c|c|c|c|c|}
\hline Patient ID & Age (years) & $\begin{array}{l}\text { Sex } \\
(F=\text { female } \\
M=\text { male })\end{array}$ & Diagnosis & Stage & Tumor size $(\mathrm{cm})$ & $\begin{array}{l}\text { Cervial lymph } \\
\text { node metastasis }\end{array}$ & $\begin{array}{l}\text { Distant } \\
\text { metastases }\end{array}$ \\
\hline \multicolumn{8}{|c|}{ Patients $<45$ years } \\
\hline P57 & 25.50 & $\mathrm{~F}$ & PTC & I & 0.6 & None & NO \\
\hline P64 & 41.71 & $\mathrm{~F}$ & PTC & 1 & 0.8 & None & NO \\
\hline PI5 & 31.25 & $\mathrm{~F}$ & PTC & 1 & 0.5 & None & NO \\
\hline PIO & 34.16 & $\mathrm{~F}$ & PTC & I & 0.6 & None & NO \\
\hline P79 & 37.71 & $\mathrm{~F}$ & PTC & I & 0.7 & None & NO \\
\hline P9I & 38.63 & $\mathrm{~F}$ & PTC & I & 1.2 & Yes (VI lymph node) & NO \\
\hline PIIO & 39.80 & $\mathrm{~F}$ & PTC & I & 1.0 & None & NO \\
\hline P34 & 40.20 & $\mathrm{~F}$ & PTC & I & 0.6 & None & NO \\
\hline P66 & 40.28 & $M$ & PTC & 1 & 0.7 & None & NO \\
\hline P92 & 40.42 & $\mathrm{~F}$ & PTC & 1 & 1.0 & None & NO \\
\hline PI09 & 40.56 & $\mathrm{~F}$ & PTC & i & 0.5 & None & NO \\
\hline $\mathrm{PI}$ & 40.85 & $\mathrm{~F}$ & PTC & i & 0.7 & None & NO \\
\hline P94 & 41.15 & $\mathrm{~F}$ & PTC & 1 & 0.7 & None & NO \\
\hline PI08 & 41.30 & $\mathrm{~F}$ & PTC & i & 1.6 & Yes (VI lymph node) & NO \\
\hline P95 & 41.39 & $M$ & PTC & I & 1.2 & None & NO \\
\hline P68 & 41.46 & $\mathrm{~F}$ & PTC & 1 & 0.8 & None & NO \\
\hline PI06 & 41.58 & $M$ & PTC & i & 1.5 & None & NO \\
\hline P96 & 41.66 & $\mathrm{~F}$ & PTC & 1 & 0.7 & None & NO \\
\hline P30 & 41.82 & $\mathrm{~F}$ & PTC & 1 & 1.0 & None & NO \\
\hline P39 & 42.05 & $\mathrm{~F}$ & PTC & 1 & 1.0 & None & NO \\
\hline P4I & 42.20 & $\mathrm{~F}$ & PTC & 1 & 1.8 & None & NO \\
\hline P89 & 42.31 & $M$ & PTC & i & 0.7 & None & NO \\
\hline P48 & 42.45 & $M$ & PTC & 1 & 1.2 & None & NO \\
\hline P98 & 42.68 & $\mathrm{~F}$ & PTC & 1 & 1.5 & None & NO \\
\hline $\mathrm{PII3}$ & 42.80 & $\mathrm{~F}$ & PTC & I & 0.9 & None & NO \\
\hline P97 & 43.40 & $\mathrm{~F}$ & PTC & 1 & 1.0 & None & NO \\
\hline P29 & 43.72 & $\mathrm{~F}$ & PTC & 1 & 1.6 & Yes (VI lymph node) & NO \\
\hline $\mathrm{P} 90$ & 44.26 & $M$ & PTC & 1 & 0.8 & None & NO \\
\hline P50 & 44.54 & $\mathrm{~F}$ & PTC & 1 & 0.7 & None & NO \\
\hline P56 & 44.98 & $\mathrm{~F}$ & PTC & i & 0.7 & None & NO \\
\hline \multicolumn{8}{|c|}{ Patients $>45$ years } \\
\hline P28 & 45.52 & $\mathrm{~F}$ & PTC & 1 & 0.8 & None & NO \\
\hline P27 & 45.66 & $\mathrm{~F}$ & PTC & II & 2.8 & None & NO \\
\hline P5I & 46.05 & $\mathrm{~F}$ & PTC & 1 & 0.6 & None & NO \\
\hline P55 & 46.62 & $M$ & PTC & 1 & 0.8 & None & NO \\
\hline P72 & 46.85 & $\mathrm{~F}$ & PTC & II & 2.8 & None & NO \\
\hline P84 & 47.25 & $\mathrm{~F}$ & PTC & 1 & 0.6 & None & NO \\
\hline P33 & 48.30 & $\mathrm{~F}$ & PTC & i & 0.8 & None & NO \\
\hline $\mathrm{P} 102$ & 48.73 & $\mathrm{~F}$ & PTC & III & 3.8 & Yes (VI lymph node) & NO \\
\hline P3I & 49.14 & $\mathrm{~F}$ & PTC & 1 & 0.6 & None & NO \\
\hline P20 & 49.60 & $\mathrm{~F}$ & PTC & I & 0.9 & None & NO \\
\hline P6I & 49.91 & $M$ & PTC & 1 & 0.8 & None & NO \\
\hline P77 & 50.40 & $\mathrm{~F}$ & PTC & 1 & 0.8 & None & NO \\
\hline P2I & 52.30 & $\mathrm{~F}$ & PTC & i & 0.9 & None & NO \\
\hline P74 & 54.56 & $M$ & PTC & III & 3.5 & Yes (VI lymph node) & NO \\
\hline P62 & 56.05 & $\mathrm{~F}$ & PTC & III & 3.5 & Yes (VI lymph node) & NO \\
\hline P86 & 62.13 & $M$ & PTC & ॥ & 2.8 & None & NO \\
\hline P75 & 67.40 & $\mathrm{~F}$ & PTC & II & 2.5 & None & NO \\
\hline P76 & 68.56 & $\mathrm{~F}$ & PTC & III & 3.8 & None & NO \\
\hline
\end{tabular}

covalently coupling the appropriate antibodies to preactivated ProteinChip arrays (Ciphergen Biosystems). Antibodies (anti-human haptoglobin alpha-chain, ms1750rbh; anti-human apolipoprotein C-I, ab9106; anti-human apolipoprotein C-III, mab002-74/3) were covalently cou- pled to PS20 arrays, respectively. After blocking with BSA and washing to remove uncoupled antibodies, antibodycoated spots were incubated with $1.5 \mu \mathrm{L}$ of serum samples and $3 \mu \mathrm{L}$ of binding buffer $\left(0.1 \mathrm{M} \mathrm{Na}_{3} \mathrm{PO}_{4}, 0.5 \mathrm{M}\right.$ urea, $0.5 \%$ CHAPS, $\mathrm{pH} 7.2$ ) for $90 \mathrm{~min}$. Spots were then 
Table 6: Study population used in SELDI experiment

\begin{tabular}{llll}
\hline & PTC Patients & Controls & Total \\
\hline Training set & 60 & $40 \mathrm{~h}^{*}$ & 100 \\
Testing set & 48 & $76\left(20 \mathrm{~h}^{\left.*+56 \mathrm{p}^{* *}\right)}\right.$ & 124 \\
\hline Total & 108 & 116 & 224 \\
\hline
\end{tabular}

$h^{*}$ : Healthy controls

$\mathrm{P}^{* *}$ : Patients with benign thyroid node

washed with PBST (0.5\% Triton X-100), PBS and deionized water twice respectively before drying. SELDI-TOFMS analysis was performed on a PBS-II ProteinChip reader with CHCA as matrix.

\section{Abbreviations}

PTC: papillary thyroid carcinoma; SELDI-TOF-MS: surface-enhanced laser desorption/ionization time-of-flight mass spectroscopy; $m / z$ : mass to charge; HPLC: high Performance Liquid Chromatography; LC-MS/MS: liquid chromatography tandem mass spectrometry; MALDITOF-MS: matrix-assisted laser desorption/ionization time-of-flight mass spectroscopy; WCX: weak cation exchange; SVM: support vector machine; $\mathrm{S} / \mathrm{N}$ : signal to noise ratio; NCBI: national center for biotechnology information.

\section{Competing interests}

The authors declare that they have no competing interests.

\section{Authors' contributions}

WJX and YFQ designed the study. FYX was responsible for laboratory studies and drafted the manuscript. LQL, DR and ZQ carried out the SELDI-TOF experiments. SLN, WP and YSB participated in the purification and identification of all the biomarkers. YJK carried out the SELDI-TOF data analysis. FYZ and YHY participated in data analysis. ZS participated in the design of the study and helped to draft the manuscript. All authors read and approved the final manuscript.

\section{Acknowledgements}

This work was supported by the National natural science foundation of China (30772273). The authors wish to thank Dr. Liwei Mi and Dr. Shutang Wen for the preparation of this manuscript.

\section{References}

I. Ronckers CM, McCarron P, Ron E: Thyroid cancer and multiple primary tumors in the SEER cancer registries. Int J Cancer 2005, I I 7:28I-288.

2. Jemal A, Siegel R, Ward E, Hao Y, Xu J, Murray T, Thun MJ: Cancer statistics, 2008. CA Cancer J Clin 2008, 58:7 I-96.

3. Pelizzo MR, Merante Boschin I, Toniato A, Casal Ide E, Mian C, Rubello D, Pagetta C: Diagnosis, treatment, prognostic factors and long-term outcome in papillary thyroid carcinoma. Minerva Endocrinol 2008, 33:359-379.
4. Hayashi N, Kitaoka M: Fine-needle aspiration biopsy of the thyroid nodule: uses and limitations. Nippon Rinsho 2007, 65:2003-2007.

5. Inohara H, Segawa T, Miyauchi A, Yoshii T, Nakahara S, Raz A, Maeda M, Miyoshi E, Kinoshita N, Yoshida H, Furukawa M, Takenaka Y, Takamura $Y$, Ito $Y$, Taniguchi $N$ : Cytoplasmic and serum galectin-3 in diagnosis of thyroid malignancies. Biochem Biophys Res Commun 2008, 376:605-610.

6. Saussez S, Glinoer D, Chantrain G, Pattou F, Carnaille B, Andre S, Gabius HJ, Laurent G: Serum galectin-I and galectin-3 levels in benign and malignant nodular thyroid disease. Thyroid 2008, 18:705-7|2.

7. Asa SL: The role of immunohistochemical markers in the diagnosis of follicular-patterned lesions of the thyroid. Endocr Pathol 2005, 16:295-309.

8. Nygaard B, Frisch T, Kiss K: Thyroid papillary cancer using TPO staining. Ugeskr Laeger 2008, 170:157I.

9. Park YJ, Kwak SH, Kim DC, Kim H, Choe G, Park J, Jang HC, Park SH, Cho BY, Park SY: Diagnostic value of galectin-3, HBME-I, cytokeratin 19, high molecular weight cytokeratin, cyclin D I and p27(kip I) in the differential diagnosis of thyroid nodules. J Korean Med Sci 2007, 22:62I-628.

10. Maurya P, Meleady P, Dowling P, Clynes M: Proteomic approaches for serum biomarker discovery in cancer. Anticancer Res 2007, 27:1247-I255.

II. Tomosugi N: Discovery of disease biomarkers by ProteinChip system; clinical proteomics as noninvasive diagnostic tool. Rinsho Byori 2004, 52:973-979.

12. Luo J, Qian JH, Yu JK, Zheng S, Xie X, Lu WG: Discovery of altered protein profiles in epithelial ovarian carcinogenesis by SELDI mass spectrometry. Eur J Gynaecol Oncol 2008, 29:233-238.

13. Wang J, Zhang X, Ge X, Guo H, Xiong G, Zhu Y: Proteomic studies of early-stage and advanced ovarian cancer patients. Gynecol Oncol 2008, I I I: I II-II9.

14. Skytt A, Thysell E, Stattin P, Stenman UH, Antti H, Wikstrom P: SELDI-TOF MS versus prostate specific antigen analysis of prospective plasma samples in a nested case-control study of prostate cancer. Int J Cancer 2007, I 21:615-620.

15. Liu D, Cao L, Yu J, Que R, Jiang W, Zhou Y, Zhu L: Diagnosis of Pancreatic Adenocarcinoma Using Protein Chip Technology. Pancreatology 2008, 9: I27-135.

16. Hundt $\mathrm{S}$, Haug $\cup$, Brenner $\mathrm{H}$ : Blood markers for early detection of colorectal cancer: a systematic review. Cancer Epidemiol Biomarkers Prev 2007, 16:1935-1953.

17. Goncalves A, Bertucci F, Birnbaum D, Borg JP: Protein profiling SELDI-TOF and breast cancer: clinical potential applications. Med Sci 2007, 23:23-26.

18. Suriano R, Lin Y, Ashok BT, Schaefer SD, Schantz SP, Geliebter J, Tiwari RK: Pilot study using SELDI-TOF-MS based proteomic profile for the identification of diagnostic biomarkers of thyroid proliferative diseases. J Proteome Res 2006, 5:856-86I.

19. Moretz WH, Gourin CG, Terris DJ, Xia ZS, Liu Z, Weinberger PM, Chin E, Adam BL: Detection of papillary thyroid carcinoma with serum protein profile analysis. Arch Otolaryngol Head Neck Surg 2008, 134:198-202.

20. Somorjai RL, Dolenko B, Baumgartner R: Class prediction and discovery using gene microarray and proteomics mass spectroscopy data: curses, caveats, cautions. Bioinformatics 2003, 19:|484-|49|.

21. Wang XD, Wang JQ: A survey on support vector machines training and testing algorithms. Computer Engineering and Application 2004, 13:75-79.

22. Matheny ME, Resnic FS, Arora N, Ohno-Machado L: Effects of SVM parameter optimization on discrimination and calibration for post-procedural PCI mortality. J Biomed Inform 2007, 40:688-697.

23. Fang J, Dong $Y$, Williams TD, Lushington $\mathrm{GH}$ : Feature selection in validating mass spectrometry database search results. J Bioinform Comput Biol 2008, 6:223-240.

24. Wassell J: Haptoglobin: function and polymorphism. Clin lab 2000, 46:547-552.

25. Weinberg ED: The role of iron in cancer. Eur J Cancer Prev 1996, 5:19-36.

26. Reizenstein P: Iron, free radicals and cancer. Med Oncol Tumor Pharmacother 1991, 8:229-233. 
27. Saldova R, Royle L, Radcliffe CM, Abd Hamid UM, Evans R, Arnold JN, Banks RE, Hutson R, Harvey DJ, Antrobus R, Petrescu SM, Dwek RA, Rudd PM: Ovarian cancer is associated with changes in glycosylation in both acute-phase proteins and IgG. Glycobiology 2007, 17:1344-1356

28. Saito S, Murayama Y, Pan Y, Taima T, Fujimura T, Murayama K, Sadilek M, Egawa S, Ueno S, Ito A, Ishidoya S, Nakagawa H, Kato M, Satoh M, Endoh M, Arai Y: Haptoglobin-beta chain defined by monoclonal antibody RM2 as a novel serum marker for prostate cancer. Int J Cance 2008, I 23:633-640.

29. Nakano M, Nakagawa T, Ito T, Kitada T, Hijioka T, Kasahara A, Tajiri M, Wada Y, Taniguchi N, Miyoshi E: Site-specific analysis of N-glycans on haptoglobin in sera of patients with pancreatic cancer: a novel approach for the development of tumor markers. Int J Cancer 2008, 1 22:230 I-2309.

30. Huang HL, Stasyk T, Morandell S, Dieplinger H, Falkensammer G, Griesmacher A, Mogg M, Schreiber M, Feuerstein I, Huck CW, Stecher G, Bonn GK, Huber LA: Biomarker discovery in breast cancer serum using 2-D differential gel electrophoresis/ MALDI-TOF/TOF and data validation by routine clinical assays. Electrophoresis 2006, 27:164I-1650.

31. Song G, Ouyang G, Bao S: The activation of Akt/PKB signaling pathway and cell survival. J Cell Mol Med 2005, 9:59-7I.

32. Lavrentiadou SN, Hadzopoulou-Cladaras M, Kardassis D, Zannis VI: Binding specificity and modulation of the human ApoCIII promoter activity by heterodimers of ligand-dependent nuclear receptors. Biochemistry 1999, 38:964-975.

33. Haymart MR, Repplinger DJ, Leverson GE, Elson DF, Sippel RS, Jaume $\mathrm{JC}$, Chen $\mathrm{H}$ : Higher serum thyroid stimulating hormone level in thyroid nodule patients is associated with greater risks of differentiated thyroid cancer and advanced tumor stage. J Clin Endocrinol Metab 2008, 93:809-8I4.

Publish with Bio Med Central and every scientist can read your work free of charge

"BioMed Central will be the most significant development for disseminating the results of biomedical research in our lifetime. "

Sir Paul Nurse, Cancer Research UK

Your research papers will be:

- available free of charge to the entire biomedical community

- peer reviewed and published immediately upon acceptance

- cited in PubMed and archived on PubMed Central

- yours - you keep the copyright

Submit your manuscript here:

http://www.biomedcentral.com/info/publishing_adv.asp
BioMedcentral 